\title{
Immunohistochemical evaluation of nuclear 5-hydroxymethylcytosine (5-hmC) accurately distinguishes malignant pleural mesothelioma from benign mesothelial proliferations
}

\author{
David B. Chapel $\mathbb{1}^{1} \cdot$ Aliya N. Husain ${ }^{1} \cdot$ Thomas Krausz $^{1}$
}

Received: 19 April 2018 / Revised: 10 September 2018 / Accepted: 16 September 2018 / Published online: 12 October 2018

(c) United States \& Canadian Academy of Pathology 2018

\begin{abstract}
Accurate distinction of benign mesothelial proliferations from malignant mesothelioma remains a diagnostic challenge. Sequential use of BAP1 immunohistochemistry and CDKN2A fluorescence in situ hybridization is specific for diagnosis of mesothelioma, but fluorescence in situ hybridization is both costly and time-consuming. Early data indicate that mesothelioma shows extensive loss of nuclear 5-hydroxymethylcytosine (5-hmC). We studied 49 cases of mesothelioma (17 epithelioid mesothelioma, 22 biphasic mesothelioma, and 10 sarcomatoid mesothelioma) and 23 benign mesothelial proliferations using a 5-hmC single immunohistochemical stain, CAM5.2/5-hmC double immunohistochemical stain, and BAP1 immunohistochemistry. Estimations of extent of 5-hmC loss were made using the 5-hmC single stain and CAM5.2/5$\mathrm{hmC}$ double stain, and extent of nuclear 5-hmC loss was definitively quantitated in at least 1000 cells per case. Mean nuclear 5 -hmC loss in mesothelioma (84\%) was significantly greater than in benign mesothelial proliferations $(4 \%)(p<0.0001)$. Using 5-hmC loss in $>50 \%$ of tumor nuclei to define the diagnosis of mesothelioma, 5-hmC immunohistochemistry showed sensitivity of $92 \%$ and specificity of $100 \%$. An immunopanel including 5-hmC and BAP1 immunohistochemistry achieved sensitivity of $98 \%$ and specificity of $100 \%$. Extensive nuclear 5-hmC loss is sensitive and specific for mesothelioma in the differential diagnosis with benign mesothelial proliferations. In challenging mesothelial lesions, immunohistochemical studies showing either extensive 5-hmC loss or BAP1 loss indicate a diagnosis of mesothelioma, precluding the need for $C D K N 2 A$ fluorescence in situ hybridization in a considerable number of cases.
\end{abstract}

\section{Introduction}

Distinction of malignant mesothelioma from benign mesothelial proliferations is a persistent challenge. Careful morphological evaluation remains the mainstay of diagnosis, but architectural features of malignancy cannot be consistently assessed in small biopsy specimens, and are effectively absent in effusion cytology specimens. To assist in this challenging distinction, a number of immunophenotypic and molecular genetic markers of malignancy have

Electronic supplementary material The online version of this article (https://doi.org/10.1038/s41379-018-0159-7) contains supplementary material, which is available to authorized users.

David B. Chapel

david.chapel@uchospitals.edu

1 Department of Pathology, University of Chicago, $5841 \mathrm{~S}$. Maryland Ave, Chicago, Illinois, USA been reported over the last two decades, with variable sensitivity, specificity, and uptake into routine pathology practice [1-7].

Two markers in particular have shown sufficient specificity to be of considerable diagnostic value. Loss of nuclear BRCA1-associated protein 1 (BAP1) by immunohistochemistry has been shown to be virtually $100 \%$ specific for distinction of malignant mesothelioma from both reactive mesothelial proliferations and carcinoma [4, 5, 8-11]. However, BAP1 immunohistochemistry shows suboptimal sensitivity, with only $56 \%$ of pleural mesothelioma overall showing BAP1 loss in one large meta-analysis [12]. Furthermore, the frequency of BAP1 loss varies by histotype, occurring in about $74 \%$ of epithelioid mesothelioma but only in $7 \%$ of sarcomatoid mesothelioma [12]. Molecular studies have shown that BAP1 loss by immunohistochemistry is a reliable surrogate for genetic alterations in BAPl [11].

Homozygous deletion of $C D K N 2 A$ has also shown excellent specificity for diagnosis of mesothelioma. 
However, as with BAP1 immunohistochemistry, sensitivity is imperfect, with $C D K N 2 A$ homozygous deletion occurring in only $52-68 \%$ of pleural mesothelioma [4, 5]. Furthermore, immunohistochemistry for the protein product of $C D K N 2 A$, p16, is not a reliable surrogate for $C D K N 2 A$ homozygous deletion [5], and current practice requires fluorescence in situ hybridization studies for accurate diagnosis. Fluorescence in situ hybridization is considerably more expensive than immunohistochemistry, requires a send-out consult test at most institutions, and has a turnaround time of several days. While preliminary reports indicate that immunohistochemistry to detect deletion of an adjacent 9p21.3-related gene, MTAP, is a highly specific surrogate for $C D K N 2 A$ homozygous deletion, the sensitivity of this method is lower than the combination of BAP1 immunohistochemistry and $C D K N 2 A$ fluorescence in situ hybridization, and MTAP immunohistochemistry is not yet in widespread diagnostic use [5, 6].

Given the excellent specificity but suboptimal sensitivity of BAP1 immunohistochemistry and $C D K N 2 A$ fluorescence in situ hybridization when performed alone, these tests are typically incorporated into a stepwise diagnostic algorithm, beginning with BAP1 immunohistochemistry and proceeding to $C D K N 2 A$ fluorescence in situ hybridization in cases with retained BAP1. This algorithm for diagnosis of mesothelioma has a sensitivity of 58-93\% [4, 10] and apparent specificity of $100 \%$ [10]. While this diagnostic approach serves in the majority of cases (including applicability to small biopsy and effusion cytology specimens [7, $13,14]$, albeit with some reports of lower accuracy [12]), it fails to detect some mesothelioma cases, and incurs the increased cost and turnaround time associated with CDKN2A fluorescence in situ hybridization.

Reversible epigenetic methylation of genomic DNA is an important element of gene regulation. Suppression proceeds through methylation of genomic cytosine residues, forming 5-methylcytosine (5-mC), while increased gene expression follows demethylation. This demethylation is dependent in part on the ten-eleven translocation family of enzymes, which convert 5-mC to 5-hydroxymethylcytosine (5-hmC) [15]. Diverse malignancies, most classically glioblastoma and acute myeloid leukemia, are characterized by decreased activity of the ten-eleven translocation protein, with consequent reduction in conversion of $5-\mathrm{mC}$ to 5 -hmC and decrease in nuclear 5-hmC [16]. This is a complex area of tumor biology research, which has been recently reviewed elsewhere [17].

Intriguingly, reduced activity of the ten-eleven translocation protein and reduced nuclear $5-\mathrm{hmC}$ have also been reported in a small set of rat and human mesotheliomas, and this reduction in nuclear 5-hmC is reportedly detectable by immunohistochemistry [18]. However, this early study investigated only the principle of decreased 5-hmC expression in mesothelioma, and no work has yet examined its potential application as a diagnostic marker in routine pathology practice. Considering the shortcomings of the current diagnostic algorithm for mesothelioma described above, we hypothesized that 5-hmC immunohistochemistry could constitute a faster, cheaper, and potentially more sensitive marker for mesothelioma.

\section{Methods}

Following institutional review board approval (\#17-0056), 49 representative cases of diffuse pleural mesothelioma diagnosed between 2009 and 2013 were selected from the archives of the Department of Pathology at the University of Chicago, including epithelioid mesothelioma $(n=17)$, biphasic mesothelioma $(n=22)$, and sarcomatoid mesothelioma $(n=10)$. The cohort was deliberately enriched for cases of biphasic mesothelioma, as use of 5-hmC immunohistochemistry to confirm a diagnosis of biphasic mesothelioma was an area of particular interest at study inception. Fourteen cases of fibrous and organizing pleuritis comprising both epithelioid and spindled elements were included as benign reactive controls, matched to the mesothelioma cohort by age and sex. Date of diagnosis and date of death or last follow-up were identified for all cases, and patients without either a known date of death or at least 5 years of documented follow-up were excluded.

All hematoxylin and eosin (H\&E)-stained sections from each case were reviewed by two pathologists (DBC \& $\mathrm{ANH}$ ) for confirmation of the original diagnosis (in cases of mesothelioma), and for documentation of mitotic index, nuclear pleomorphism, and presence of concurrent morphologically benign reactive spindled mesothelial proliferation (in cases of epithelioid mesothelioma only). Any potential mesothelioma case without morphologic features diagnostic of mesothelioma was excluded.

A 5-hmC single immunohistochemical stain was performed on a representative section from a subset of cases (10 epithelioid mesothelioma, 11 biphasic mesothelioma, 10 sarcomatoid mesothelioma, 14 fibrous/organizing pleuritis) using a Leica Bond RX automatic stainer with a 15-min antigen retrieval (epitope retrieval solution I, Leica Biosystems, AR9961) and the 25-min UCH DAB-modified protocol with the primary anti-5-hmC antibody (1:1500; Active Motif, rabbit polyclonal, \#39769) on formalin-fixed paraffin-embedded tissue. Antigen-antibody binding was detected with Bond polymer refine detection (Leica Biosystems, DS9800).

A CAM5.2/5-hmC double immunohistochemical stain was performed on a representative section from all cases using a Leica Bond RX automatic stainer, with 20-min antigen retrieval (epitope retrieval solution I, Leica 
Biosystems, AR9961) and the 25-min DS no post primary DAB Refine protocol first with anti-5-hmC antibody (1:1500; Active Motif, rabbit polyclonal, \#39769) on formalin-fixed paraffin-embedded tissue. Antigen-antibody binding was detected with Bond polymer refine detection (Leica Biosystems, DS9800). The 25-min HTRC Red refine protocol was performed second with anti-cytokeratin antibody (1:100; CAM5.2, BD Biosciences, mouse monoclonal, \#349205). Antigen-antibody binding was detected with Bond red polymer refine detection (Leica Biosystems, DS9390). BAP1 immunohistochemistry was performed on the same representative section from each case, using a previously reported protocol [19].

The extent of 5-hmC loss in mesothelioma tumor cells was then estimated by two pathologists at a multiheaded scope, using the 5-hmC single stain and CAM5.2/5-hmC double stain. After these estimations, the extent of 5-hmC loss was definitively quantitated in all mesothelioma cases by counting at least 1000 CAM5.2-positive tumor cells on each CAM5.2/5-hmC double-stained slide. In cases of biphasic mesothelioma, 1000 cells were counted from both the epithelioid and the sarcomatoid elements. In cases with morphologically benign, reactive spindled mesothelial proliferation adjacent to epithelioid mesothelioma, 1000 cells were counted in both the malignant and the morphologically benign elements. In control cases of fibrous/organizing pleuritis, 1000 cells were counted, with 5-hmC loss in the spindled and epithelioid mesothelial elements quantitated together.

Loss of 5-hmC was defined by complete loss of nuclear staining in a given cell, in the presence of an internal positive control (i.e., adjacent mesothelioma tumor cells with retained 5-hmC, or nearby stromal cells with nuclear 5$\mathrm{hmC}$ ). At each phase of estimation or quantitation, the evaluating pathologist was blinded to the diagnosis and the results of previous estimations for the case. BAP1 expression was scored as either retained or lost. Loss of BAP1 was defined by complete absence of BAP1 staining in tumor cell nuclei, in the presence of an internal positive control (BAP1-positive stromal or inflammatory cells).

Extent of 5-hmC loss in mesothelioma cases was compared to loss in benign control tissue (including cases of fibrous/organizing pleuritis and morphologically benign reactive spindled mesothelial proliferation adjacent to foci of epithelioid mesothelioma), and to grade in epithelioid mesothelioma cases, using analysis of variance. The relationship between extent of nuclear 5-hmC loss and survival was assessed using Pearson correlation. The Bland-Altman method was used to compare estimation of 5-hmC loss using the 5-hmC single stain, estimation of 5-hmC loss using the CAM5.2/5-hmC double stain, and definitive quantitation of 5-hmC loss using the CAM5.2/5-hmC double stain.

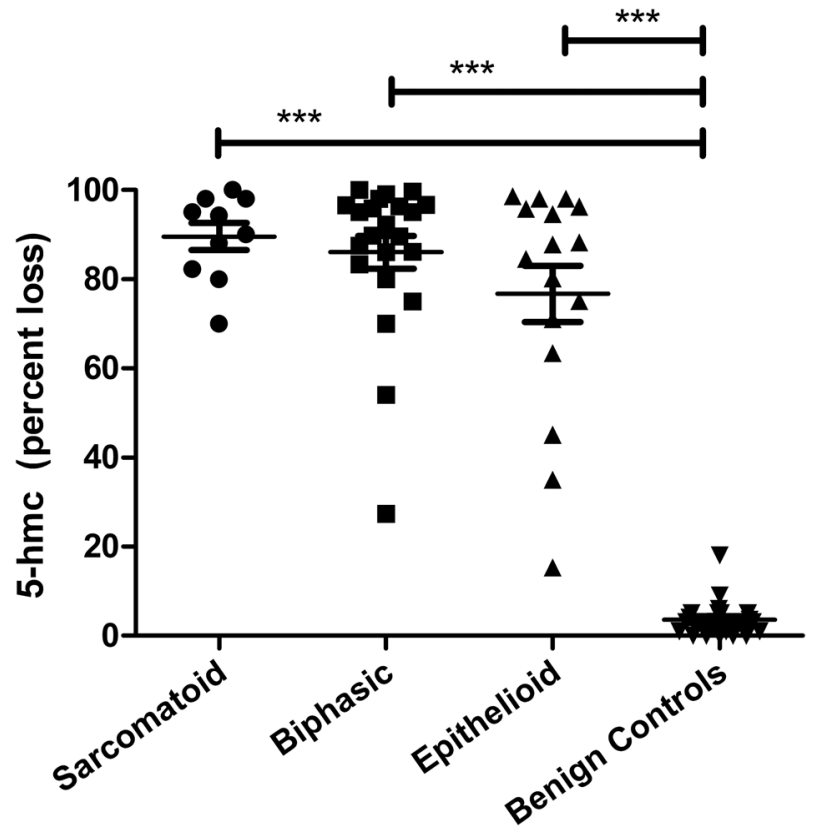

Fig. 1 Extent of 5-hmC loss is significantly greater in epithelioid, biphasic, and sarcomatoid mesothelioma, when compared to benign mesothelial proliferations $(* * * p<0.0001)$. There is no significant difference between extent of 5 -hmC loss in the subtypes of mesothelioma

\section{Results}

The mesothelioma cohort included 17 cases of epithelioid mesothelioma, 22 cases of biphasic mesothelioma, and 10 cases of sarcomatoid mesothelioma (total $n=49$ ). Fourteen cases of fibrous/organizing pleuritis were included as benign controls. Additionally, nine cases of epithelioid mesothelioma had adjacent morphologically benign reactive spindled mesothelial proliferations, for a total of 23 benign control mesothelial proliferations. The mesothelioma and benign control cohorts did not differ significantly in age or sex. Median overall survival decreased significantly from 25 months for epithelioid mesothelioma to 13 months for biphasic mesothelioma and 9 months for sarcomatoid mesothelioma. Median survival was not reached in cases of fibrous/organizing pleuritis (average follow-up of 65 months; 4 of 14 patients dead, all of non-mesothelioma causes).

Mean quantitated $5-\mathrm{hmC}$ loss was significantly greater in mesothelioma (84\%) than in benign reactive mesothelial proliferations $(4 \%)(p<0.0001)$ (Figs 1-3). Mean quantitated 5-hmC loss did not differ significantly between epithelioid (77\%), biphasic (86\%), and sarcomatoid (90\%) mesothelioma $(p=0.41)$. Mean quantitated $5-\mathrm{hmC}$ loss was significantly greater in grade 3 epithelioid mesothelioma (97\%) than in grade 1 and 2 epithelioid mesothelioma $(68 \%)(p=0.009)$. Greater 5 -hmC loss was weakly but 


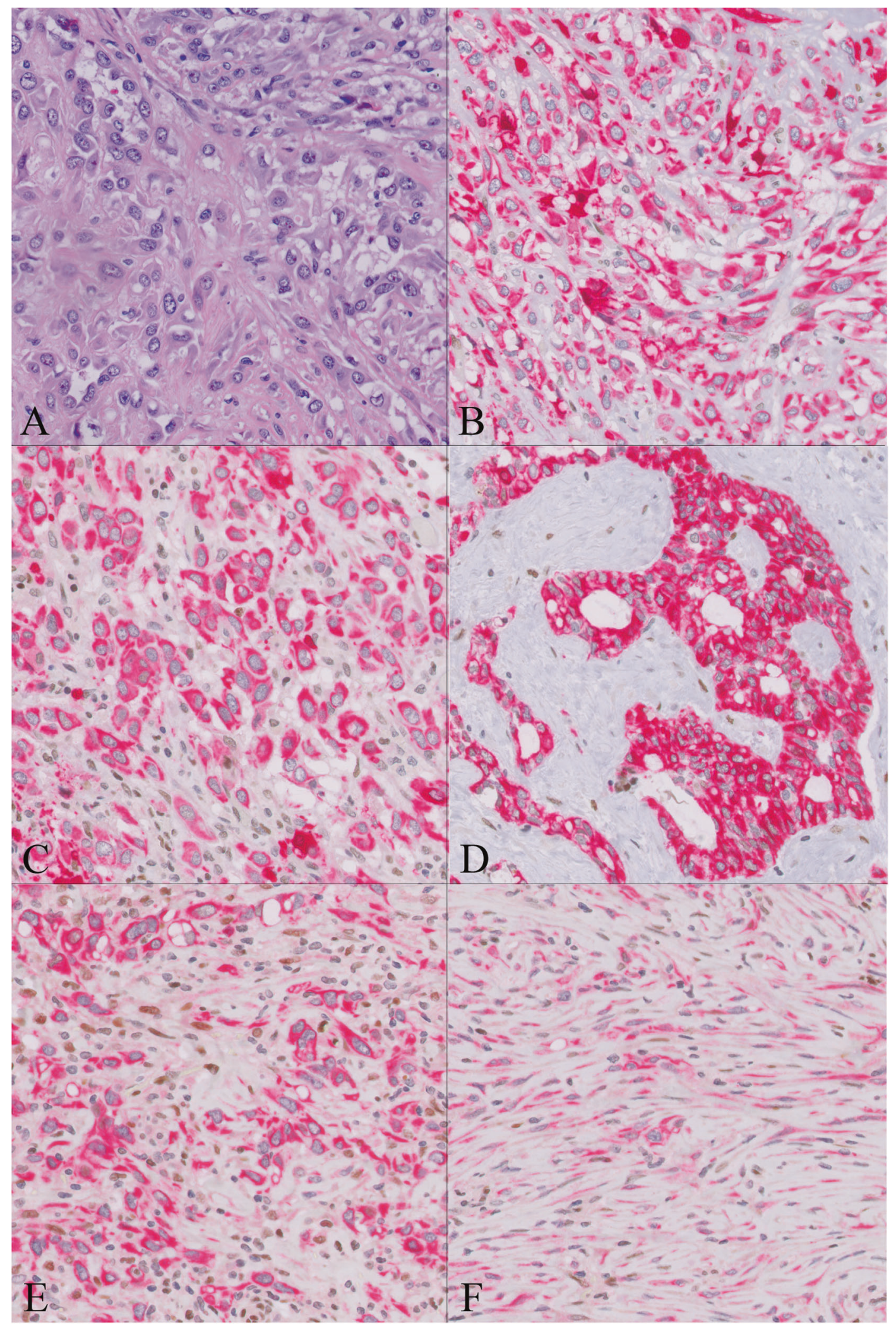

Fig. 2 a The sarcomatoid component of a biphasic mesothelioma (H\&E, original magnification 200x). On CAM5.2/5-hmC double stain (CAM5.2-red chromogen; 5-hmC - brown chromogen), both sarcomatoid tumor cells $(\mathbf{b}, \mathbf{c})$ and epithelioid tumor cells (d) express cytoplasmic keratin and show effectively $100 \%$ loss of nuclear 5-hmC (original magnification 200x). In a separate case of sarcomatoid mesothelioma (e, f), tumor cells similarly show cytoplasmic staining for keratins, with extensive loss of nuclear 5-hmC (original magnification 200x). In both cases, non-tumor inflammatory and spindle cells provide a positive internal control 


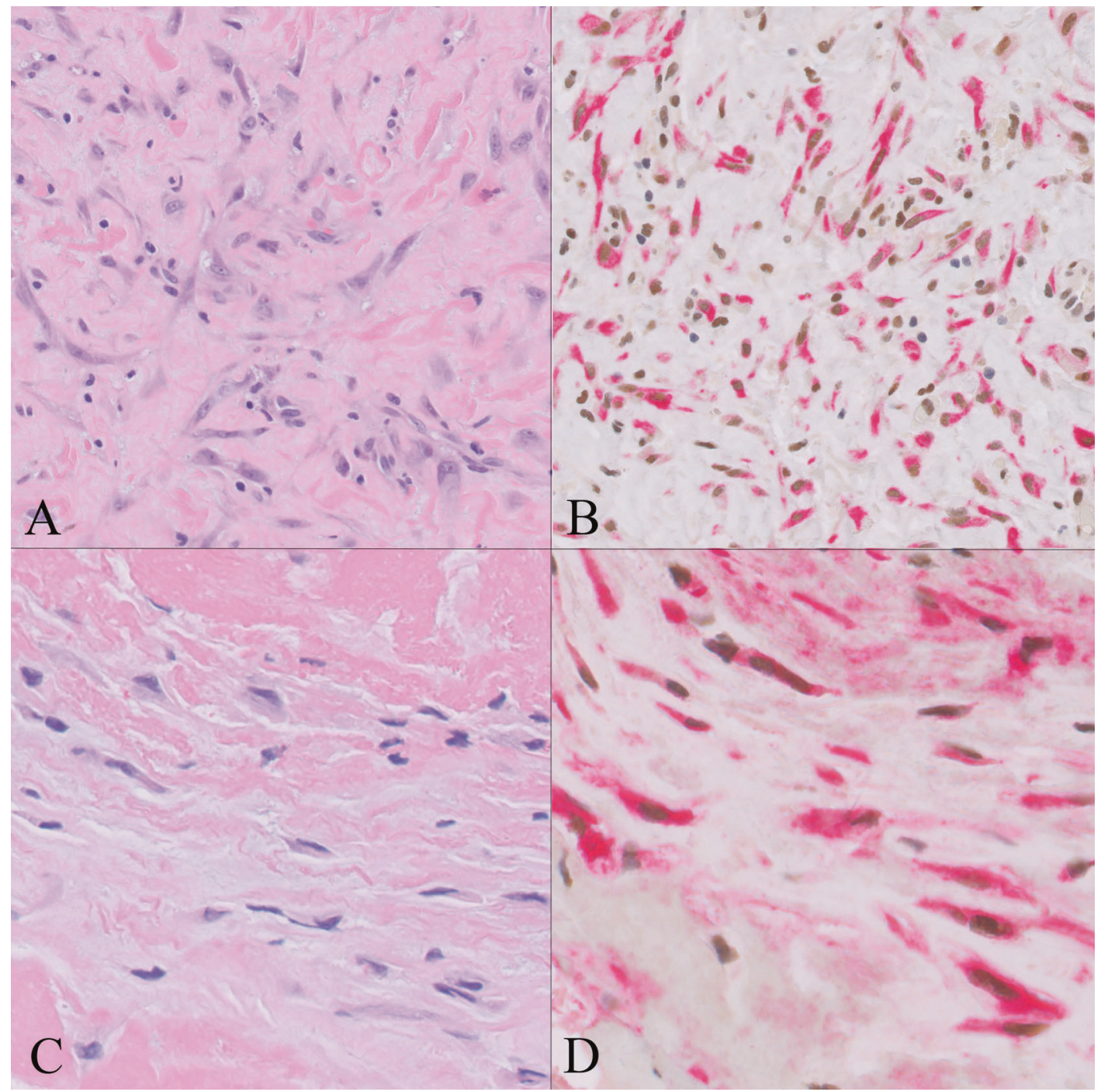

Fig. 3 a Benign pleuritis shows spindled cells haphazardly arranged in a fibrous background (H\&E, original magnification 200x). b On CAM5.2/5-hmC double stain (CAM5.2 —red chromogen; 5-hmCbrown chromogen; original magnification 200x), spindled mesothelial cells show cytoplasmic keratins and broad retention of nuclear 5-hmC
(9\% quantitated nuclear 5-hmC loss in mesothelial cells). c, d In a separate case of benign pleuritis, spindled cells in fibrous stroma show near-total retention of nuclear 5-hmC (1\% quantitated nuclear 5-hmC loss in mesothelial cells) (c: H\&E, original magnification 400x; d: CAM5.2/5-hmC double stain, original magnification $400 \times$ ) significantly associated with shorter survival among all mesothelioma cases $(r=-0.32, p=0.028)$.

A receiver-operator characteristics curve for use of CAM5.2/5-hmC double stain in diagnosis of mesothelioma shows an area under the curve of 0.999 (95\% CI 0.996-1.00). Defining malignancy by $>50 \%$ 5-hmC loss, sensitivity is $92 \%$ (95\% CI $80.0-98 \%$ ) and specificity is $100 \%$ (95\% CI $85-100 \%)$. Using a $50 \%$ cutoff, there were two "borderline" cases, with one case falling between 40 and $50 \%$ nuclear 5 -hmC loss, and one case falling between 50 and $60 \% 5$-hmC loss. For comparison, selecting a higher cutoff of $>80 \% 5$-hmC loss to define mesothelioma, sensitivity is $73 \%(95 \%$ CI $58-85 \%)$ and specificity $100 \%$ (95\% CI 85-100\%) (Supplemental Fig. 1).
Nuclear BAP1 was lost in 14 of $17(82 \%)$ epithelioid mesothelioma, 11 of $22(50 \%)$ biphasic mesothelioma, and 0 of 10 sarcomatoid mesothelioma. All benign reactive mesothelial proliferations showed retention of BAP1. Only 1 of $49(2 \%)$ mesothelioma cases showed both < $50 \%$ quantitated $5-\mathrm{hmC}$ loss and nuclear BAP1 retention. When considered together as an immunopanel, CAM5.2/ 5-hmC double stain (using $>50 \%$ loss as criterion for malignancy) and BAP1 immunohistochemistry had sensitivity of $98 \%$ and specificity of $100 \%$ for diagnosis of mesothelioma. An immunopanel including the 5-hmC single stain and BAP1 immunohistochemistry failed to diagnose 3 of $31(10 \%)$ tested mesothelioma cases (sensitivity $90 \%$, specificity $100 \%$ ). No benign control cases 
Estimation with 5-hmC single IHC stain,

vs quantitation with CAM5.2 / 5-hme double IHC stain
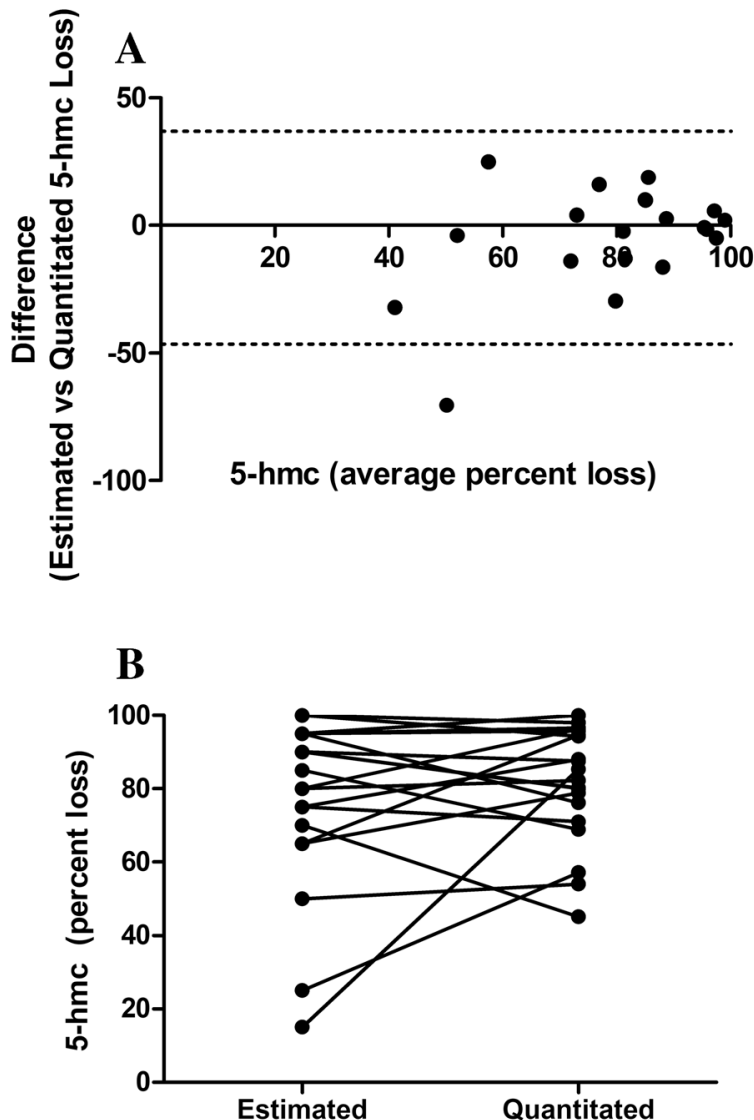

Fig. 4 Bland-Altman analyses for mesothelioma cases. a, b Estimation with the 5-hmC single stain underestimated 5-hmC loss by up to 70 percentage points and overestimated by up to 25 percentage points, compared to definitive 5-hmC quantitation using a CAM5.2/5-hmC double stain ( $95 \%$ confidence interval: -46 percentage points to +37

were erroneously diagnosed as mesothelioma by either of these immunopanels.

Considering only the nine cases of epithelioid mesothelioma with an adjacent morphologically benign reactive spindled mesothelial proliferation, the mean quantitated 5$\mathrm{hmC}$ loss was $68 \%$ (range 15-100\%) in the malignant epithelioid elements and $3.5 \%$ (range $0-5 \%$ ) in the benign reactive spindled elements $(p=0.0005)$. Across these nine cases, the average difference in quantitated 5 -hmC loss between malignant epithelioid and adjacent benign reactive spindled elements was 64 percentage points, and the smallest difference in a single case was 15 percentage points.

Compared to gold-standard quantitation of 5-hmC loss by CAM5.2/5-hmC double stain, estimation of $5-\mathrm{hmC}$ loss using the 5-hmC single stain ranged from underestimation by 70 percentage points to overestimation by 25 percentage points ( $95 \% \mathrm{CI}$, underestimation by 46 percentage points to
Estimation with CAM5.2 / 5-hmC double IHC stain, vs quantitation with CAM5.2 / 5-hme double IHC stain
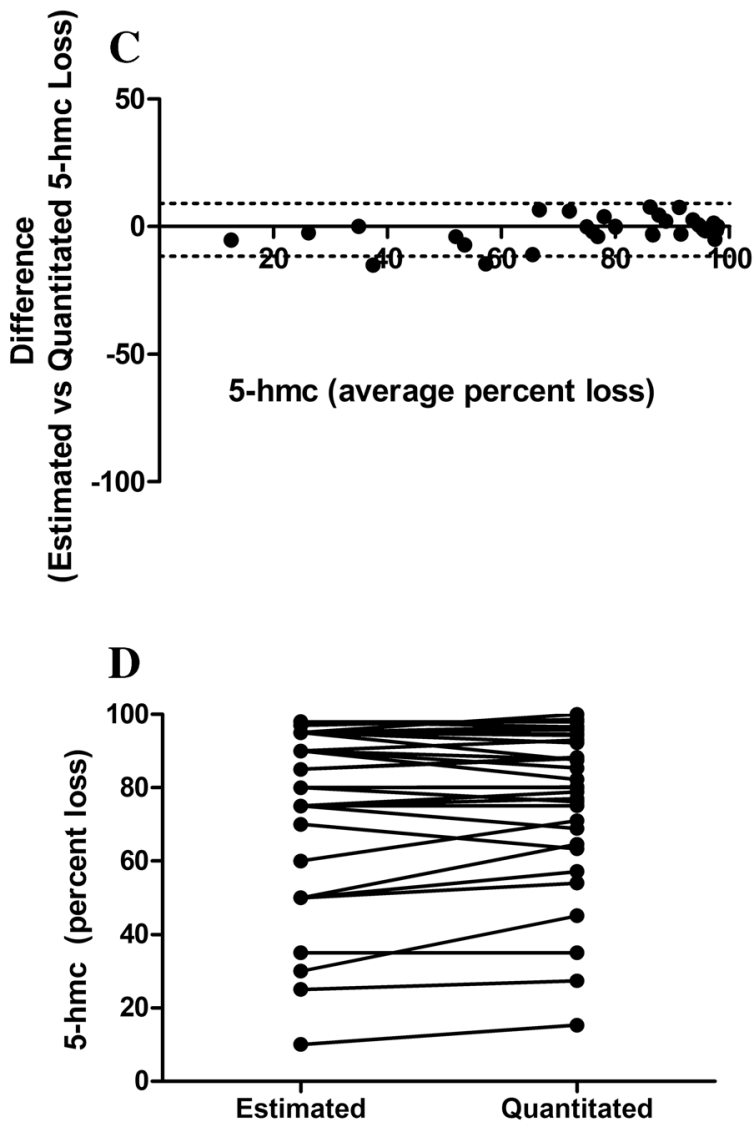

percentage points). c, d Estimation with the CAM5.2/5-hmC double stain underestimated $5-\mathrm{hmC}$ loss by up to 15 percentage points and overestimated by up to 8 percentage points, compared to definitive 5hmC quantitation using a CAM5.2/5-hmC double stain $(95 \%$ confidence interval: -12 percentage points to +9 percentage points)

overestimation by 37 percentage points) (Fig. 4a, b). Using the same quantitated gold standard, accuracy of estimated 5$\mathrm{hmC}$ loss using the CAM5.2/5-hmC double stain ranged from underestimation by 15 percentage points to overestimation by 8 percentage points $(95 \% \mathrm{CI}$, underestimation by 12 percentage points to overestimation by 9 percentage points) (Fig. 4c, d).

\section{Discussion}

Here we report that quantitation of 5-hmC loss accurately distinguishes mesothelioma from benign mesothelial proliferations, and that estimation of 5-hmC loss with a CAM5.2/5-hmC double stain yields sensitivity and specificity comparable to outright quantitation. These findings build on previous research reporting decreased expression of the ten-eleven translocation protein and decreased 


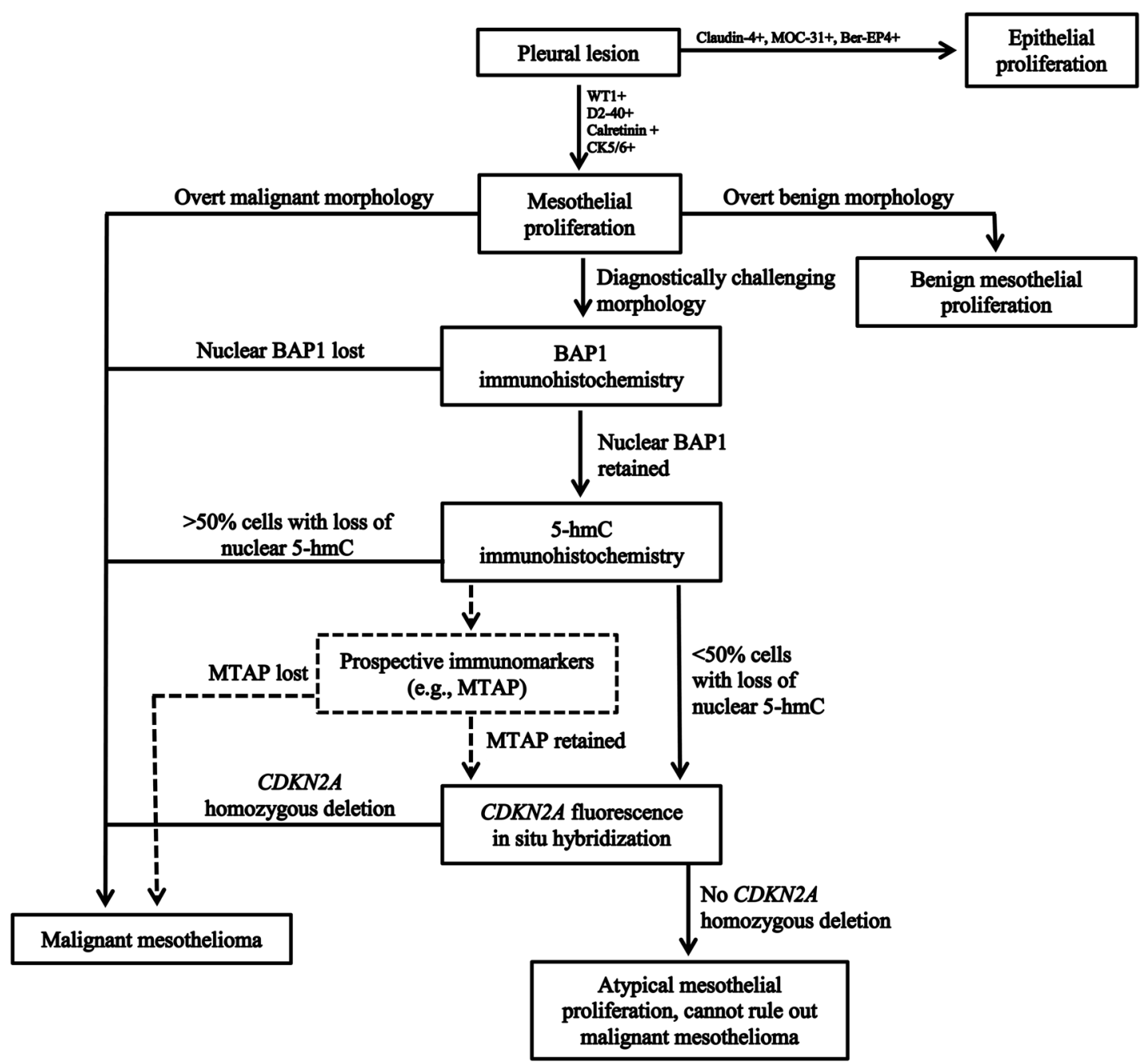

Fig. 5 Modification of the currently accepted algorithm for diagnosis of challenging mesothelial proliferations may eliminate need for $C D K N 2 A$ fluorescence in situ hybridization in a subset of cases.
Inclusion of novel immunomarkers, such as MTAP [5], may further streamline diagnosis nuclear 5-hmC in a small set of rat and human mesotheliomas [18]. The current study is an important step in the validation of 5-hmC immunohistochemistry as a useful diagnostic test for mesothelioma in routine pathology practice.

Our data show both a statistically significant and a diagnostically relevant difference in extent of 5-hmC loss between mesothelioma and benign reactive mesothelial proliferations. This indicates that 5-hmC immunohistochemistry distinguishes mesothelioma from benign mesothelial proliferations, providing a valuable adjunct in diagnostically challenging cases. Further, an immunopanel comprising BAP1 immunohistochemistry and CAM5.2/5hmC double stain has specificity of $100 \%$ and sensitivity up to $98 \%$ (depending on the 5 -hmC cutoff used to define mesothelioma, as well as the prevalence of BAP1 loss in the mesothelioma cohort). This is appreciably higher sensitivity than the $\sim 60-90 \%$ previously reported for a diagnostic algorithm proceeding from BAP1 immunohistochemistry to $C D K N 2 A$ fluorescence in situ hybridization [4, 10, 20], and suggests a modification to the currently accepted diagnostic approach for challenging mesothelial proliferations, with insertion of 5-hmC immunohistochemistry early in the diagnostic workup (Fig. 5). To wit, using the accepted algorithm and proceeding directly to $C D K N 2 A$ fluorescence in situ hybridization in BAP1-retained cases, fluorescence in situ hybridization studies may have been necessary in up to 22 of our 49 mesothelioma cases, while addition of the CAM5.2/5-hmC double stain to the algorithm reduces the need for $C D K N 2 A$ fluorescence in situ hybridization to a single case with both $<50 \%$ nuclear 5 -hmC loss and BAP1 retention (Fig. 6). Undoubtedly, additional such cases of mesothelioma will arise, in which a BAP1 \& 5-hmC immunopanel does not secure the diagnosis of malignancy; 


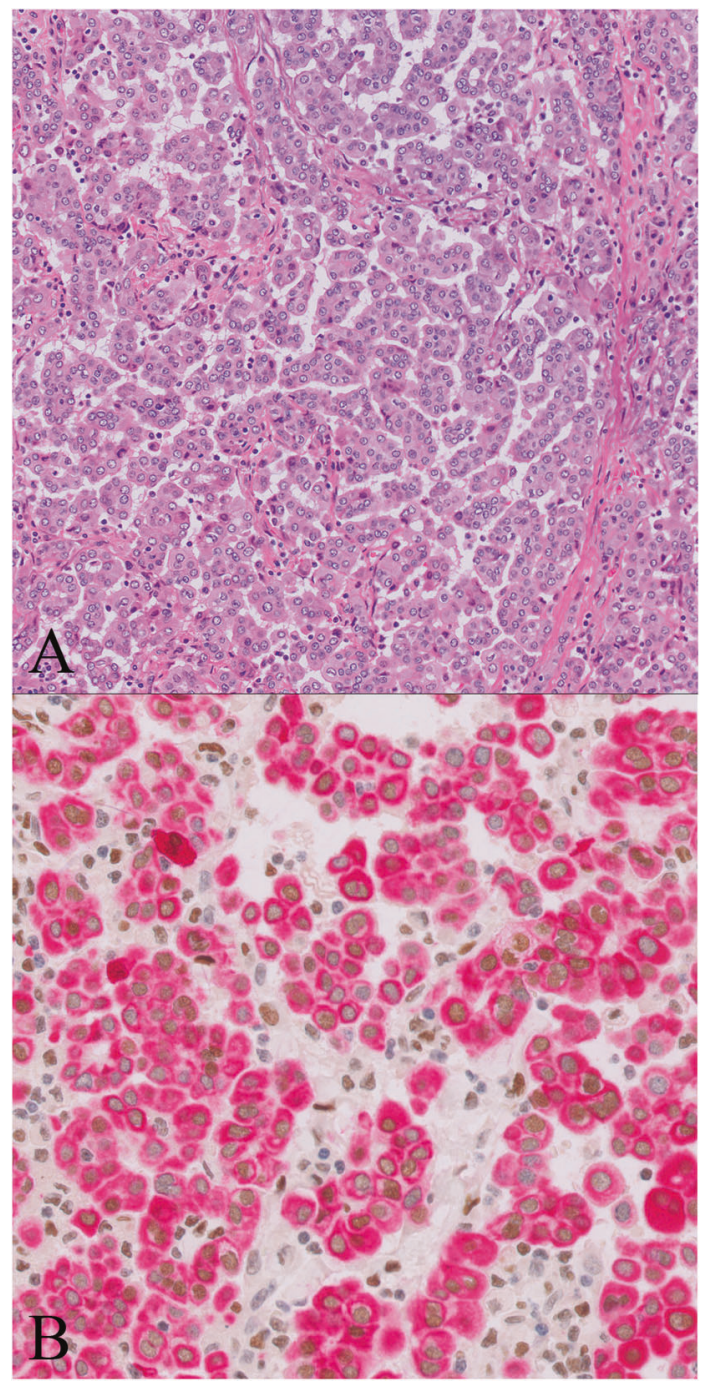

Fig. 6 a This unusual case comprises low-grade epithelioid malignant mesothelioma with micropapillary architecture (H\&E, original magnification 100x). b On CAM5.2/5-hmC double stain (CAM5.2-red chromogen; 5-hmC - brown chromogen; original magnification 200x), there is loss of $5-\mathrm{hmC}$ in just $35 \%$ of tumor nuclei-insufficient for a diagnosis of malignant mesothelioma

in such cases, $C D K N 2 A$ fluorescence in situ hybridization remains an excellent independent diagnostic test, with the potential for new immunohistochemical markers, such as MTAP, to enter the diagnostic algorithm, streamlining diagnosis and further reducing the need for fluorescence in situ hybridization. (As an aside, this particular case was also negative for $C D K N 2 A$ homozygous deletion on clinical fluorescence in situ hybridization, highlighting the existence of rare cases in which no currently available ancillary tests are able to definitively confirm a diagnosis of mesothelioma.).

We also identify a significant difference in 5-hmC loss between epithelioid mesothelioma and morphologically benign reactive mesothelial proliferations in the same specimen. These findings strongly suggest that, in cases with a component of overt epithelioid mesothelioma adjacent to an atypical spindled mesothelial proliferation, quantitation of 5-hmC loss in the spindled mesothelial population can help to classify it as benign or malignant, thus distinguishing true biphasic mesothelioma from epithelioid mesothelioma with an adjacent benign mesothelial reaction.

The CAM5.2/5-hmC double stain provides an intuitive and effective method for estimating 5-hmC loss, and permits easy identification of internal positive controls for 5hmC (Figs 7, 8). Bland-Altman analysis confirms that the CAM5.2/5-hmC double stain is more accurate than the 5$\mathrm{hmC}$ single stain for estimation of 5-hmC loss, and that the double stain shows greater sensitivity for diagnosis of mesothelioma. An immunopanel including BAP1 immunohistochemistry and the CAM5.2/5-hmC double stain would miss only $2 \%$ of mesothelioma cases, while $\sim 10 \%$ of mesothelioma cases could be missed by an immunopanel of BAP1 immunohistochemistry and the 5-hmC single stain (see Fig. 4b). Importantly, the specificity of both the 5-hmC single stain and the CAM5.2/5-hmC double stain appears to be excellent, and no benign cases would have been misclassified as malignant using either immunopanel. These data suggest that the CAM5.2/5-hmC double stain should be employed where possible. Although routine implementation of a double immunostain may constitute a logistical hurdle for some labs, it has been our experience that modern automated immunohistochemical processors have significantly decreased the complexity, and thus increased the accessibility, of this technique (unpublished observations). In settings where a double immunostain is not feasible, the 5-hmC single stain still remains a useful option, as it appears highly specific and permits accurate diagnosis of mesothelioma in many cases (including in some BAP1-retained cases). Additional studies of reproducibility and interobserver agreement using the 5-hmC single stain would be instructive.

In addition to apparent diagnostic utility, our findings may shed some light on the biology of mesothelioma. We find that high-grade (grade 3) epithelioid mesothelioma has significantly greater 5 -hmC loss than low-grade (grade 1 \& 2) epithelioid mesothelioma, indicating that nuclear grade correlates to a decrease in DNA demethylation. This appears concordant with previous work showing a strong inverse correlation between cell proliferation and nuclear 5hmC content [21, 22]. Negative correlation among mesothelioma patients between $5-\mathrm{hmC}$ loss and overall survival further indicates an association between 5-hmC loss and aggressive tumor biology.

Although this study shows a promising role for $5-\mathrm{hmC}$ immunohistochemistry in diagnosis of challenging mesothelial proliferations, it also raises a number of new questions. First, we studied resection specimens, with the diagnosis of mesothelioma established by accepted 


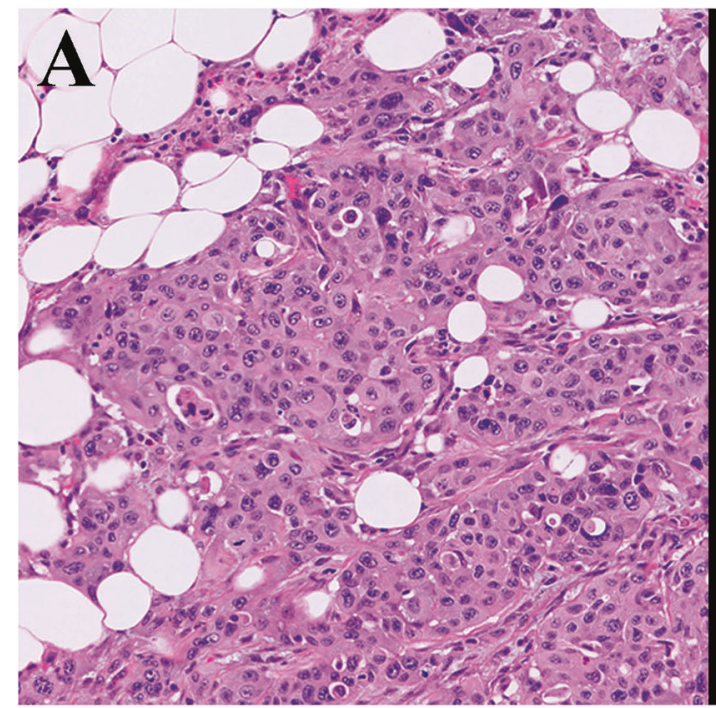

Fig. 7 a High-grade epithelioid mesothelioma with solid growth (H\&E, original magnification 200x). b On CAM.2/5-hmC double stain, tumor cells show strong cytoplasmic staining for cytokeratins

morphologic features of malignancy. Studies of 5-hmC immunohistochemistry for diagnosis in small biopsy and effusion cytology specimens are needed. In these subsequent studies, particular attention should be paid to specificity, in order to better determine the optimal 5-hmC cutoff for confidently designating a malignant tumor. Further, studies to formally pilot our proposed diagnostic algorithm, including $C D K N 2 A$ fluorescence in situ hybridization when indicated, are needed to better validate this approach (with the inclusion of additional novel markers, where appropriate). Finally, work examining additional 5hmC-related epigenetic and metabolic markers (including 5$\mathrm{mC}$, ten-eleven translocation, isocitrate dehydrogenase, succinate dehydrogenase, and fumarate dehydrogenase) may reveal a diagnostic role for these markers, and may further elucidate the interplay of genetic and epigenetic alterations in mesothelioma pathogenesis.

We observe that the prevalence of BAP1 loss in our epithelioid mesothelioma cohort $(14 / 17,82 \%)$ is at the higher end of what is considered typical for pleural epithelioid mesothelioma [12]. However, the cases in our cohort were selected without respect to BAP1 status, and the prevalence of BAP1 loss in the current cohort is consistent with previous studies of epithelioid pleural mesothelioma at our institution [23] and falls within the overall range of values reported by others (i.e., 74\% of epithelioid mesothelioma cases with BAP1 loss in one large meta-analysis) [12]. The biological roles of the BAP1 deubiquitinase enzyme and the ten-eleven translocation -5 -hmC system of epigenetic regulation are not known to be related, so the rate of BAP1 loss is not expected to impact 5-hmC loss in a given cohort. However, the somewhat higher rate of BAP1 loss in our cohort may result in slight overestimation of the

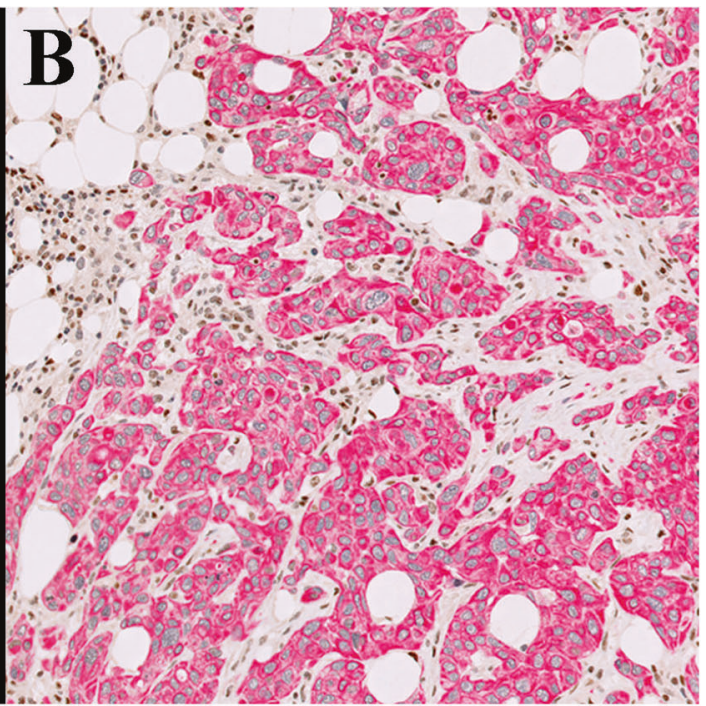

(red chromogen), but near-total loss of nuclear 5-hmC (brown chromogen). Background stromal and inflammatory cells show retained 5$\mathrm{hmC}$ (original magnification 200x)

sensitivity of a combined BAP1 +5-hmC immunopanel, compared to what may be identified in subsequent studies.

Finally, it bears mentioning that our study addresses the distinction between benign and malignant mesothelial proliferations, and should not be conflated with the challenging distinction of mesothelioma from primary or metastatic carcinoma. Although we have not studied 5-hmC loss in carcinoma, published work indicates that $5-\mathrm{hmC}$ loss is a feature of diverse malignancies [15-17]. Accordingly, we anticipate that at least some carcinomas would show extensive 5-hmC loss, and 5-hmC immunohistochemistry should not be used in the distinction of mesothelioma from carcinoma.

In conclusion, our findings indicate a promising role for 5-hmC immunohistochemistry in the diagnosis of challenging mesothelial proliferations, with excellent sensitivity and specificity for distinguishing benign proliferations from malignant mesothelioma. Use of a CAM5.2/5-hmC double stain permits highly accurate estimation of 5-hmC loss. Additional studies to investigate application of this technique to small biopsy and effusion cytology specimens are warranted, to more precisely define its diagnostic role in routine pathology practice.

Acknowledgements The authors wish to acknowledge the University of Chicago Human Tissue Resource Center for their assistance in the creation, optimization, and implementation of the CAM5.2/5-hmC double immunohistochemical stain used in this work.

\section{Compliance with ethical standards}

Conflict of interest The authors declare that they have no conflict of interest. 


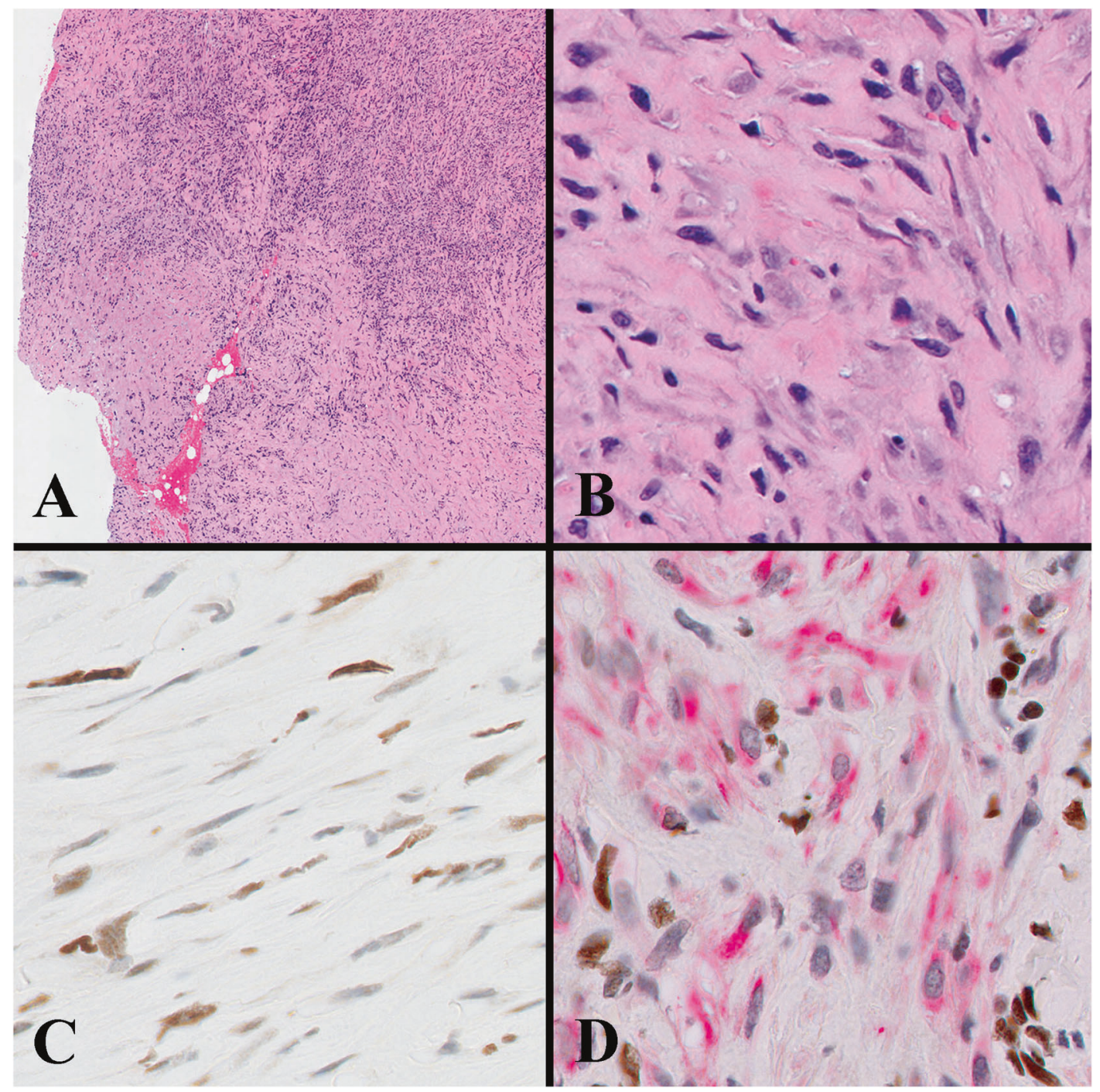

Fig. 8 a Sarcomatoid mesothelioma (H\&E, original magnification $20 \times)$. b It is difficult to distinguish mesothelial tumor cells from reactive fibroblasts (H\&E, original magnification 400x). c Some spindled cells retain nuclear 5-hmC, while others do not. Because of the difficulty in typing individual cells, it is difficult to quantitate extent of 5-hmC loss in mesothelial tumor cells $(5-\mathrm{hmC}$ single stain,

\section{References}

1. Attanoos RL, Griffin A, Gibbs AR. The use of immunohistochemistry in distinguishing reactive from neoplastic mesothelium. A novel use for desmin and comparative evaluation with epithelial membrane antigen, p53, platelet-derived growth factorreceptor, P-glycoprotein and Bcl-2. Histopathology. 2003;43:231-8.

2. Kuperman M, Florence RR, Pantanowitz L, et al. Distinguishing benign from malignant mesothelial cells in effusions by Glut-1, EMA, and Desmin expression: an evidence-based approach. Diagn Cytopathol. 2013;41:131-40.

3. Minato H, Kurose N, Fukushima M, et al. Comparative immunohistochemical analysis of IMP3, GLUT1, EMA, CD146, and desmin for distinguishing malignant mesothelioma from reactive mesothelial cells. Am J Clin Pathol. 2014;141:85-93. original magnification 400x). d Application of a CAM5.2/5-hmC double stain shows that keratin-positive mesothelial tumor cells show near-total loss of nuclear 5-hmC, while those cells with retained 5$\mathrm{hmC}$ represent keratin-negative fibroblastic, inflammatory, or endothelial cells (keratin — red chromogen; 5-hmC-brown chromogen; original magnification $400 \times$ )

4. Sheffield BS, Hwang HC, Lee AF, et al. BAP1 immunohistochemistry and p16 FISH to separate benign from malignant mesothelial proliferations. Am J Surg Pathol. 2015;39:977-82.

5. Hida T, Hamasaki M, Matsumoto $S$, et al. Immunohistochemical detection of MTAP and BAP1 protein loss for mesothelioma diagnosis: comparison with 9p21 FISH and BAP1 immunohistochemistry. Lung Cancer. 2017;104:98-105.

6. Kinoshita Y, Hida T, Hamasaki M, et al. A combination of MTAP and BAP1 immunohistochemistry in pleural effusion cytology for the diagnosis of mesothelioma. Cancer Cytopathol. 2018;126:54-63.

7. Bruno R, Ali G, Fontanini G. Molecular markers and new diagnostic methods to differentiate malignant from benign mesothelial pleural proliferations: a literature review. J Thorac Dis. 2018;10: S342-S352. 
8. Andrici J, Parkhill TR, Jung J, et al. Loss of expression of BAP1 is very rare in non-small cell lung carcinoma. Pathology . 2016;48:336-40.

9. Carbone M, Shimizu D, Napolitano A, et al. Positive nuclear BAP1 immunostaining helps differentiate non-small cell lung carcinomas from malignant mesothelioma. Oncotarget. 2016;7:59314-21.

10. Hida T, Hamasaki M, Matsumoto S, et al. BAP1 immunohistochemistry and p16 FISH results in combination provide higher confidence in malignant pleural mesothelioma diagnosis: ROC analysis of the two tests. Pathol Int. 2016;66:563-70.

11. Nasu M, Emi M, Pastorino S, et al. High incidence of somatic BAP1 alterations in sporadic malignant mesothelioma. J Thorac Oncol. 2015;10:565-76.

12. Wang LM, Shi ZW, Wang JL, et al. Diagnostic accuracy of BRCA1-associated protein 1 in malignant mesothelioma: a metaanalysis. Oncotarget. 2017;8:68863-72.

13. Cozzi I, Oprescu FA, Rullo E, et al. Loss of BRCA1-associated protein 1 (BAP1) expression is useful in diagnostic cytopathology of malignant mesothelioma in effusions. Diagn Cytopathol. 2018;46:9-14.

14. Walts AE, Hiroshima K, McGregor SM, et al. BAP1 immunostain and CDKN2A (p16) FISH analysis: clinical applicability for the diagnosis of malignant mesothelioma in effusions. Diagn Cytopathol. 2016;44:599-606.

15. Rasmussen KD, Helin K. Role of TET enzymes in DNA methylation, development, and cancer. Genes Dev. 2016;30:733-50.
16. Vasanthakumar A, Godley LA. 5-hydroxymethylcytosine in cancer: significance in diagnosis and therapy. Cancer Genet. 2015;208:167-77.

17. Waterfall JJ, Killian JK, Meltzer PS. The role of mutation of metabolism-related genes in genomic hypermethylation. Biochem Biophys Res Commun. 2014;455:16-23.

18. Roulois D, Deshayes S, Guilly MN, et al. Characterization of preneoplastic and neoplastic rat mesothelial cell lines: the involvement of TETs, DNMTs, and 5-hydroxymethylcytosine. Oncotarget. 2016;7:34664-87.

19. Chapel DB, Husain AN, Krausz T, et al. PAX8 expression in a subset of malignant peritoneal mesotheliomas and benign mesothelium has diagnostic implications in the differential diagnosis of ovarian serous carcinoma. Am J Surg Pathol. 2017; $41: 1675-82$.

20. Churg A, Sheffield BS, Galateau-Salle F. New markers for separating benign from malignant mesothelial proliferations: Are we there yet? Arch Pathol Lab Med. 2016;140:318-21.

21. Bachman $M$, Uribe-Lewis $S$, Yang $X$, et al. 5Hydroxymethylcytosine is a predominantly stable DNA modification. Nat Chem. 2014;6:1049-55.

22. Gackowski D, Zarakowska E, Starczak M, et al. Tissue-specific differences in DNA modifications (5-hydroxymethylcytosine, 5-formylcytosine, 5-carboxylcytosine and 5-hydroxymethyluracil) and their interrelationships. PLoS ONE. 2015;10:e0144859.

23. McGregor SM, Dunning R, Hyjek E, et al. BAP1 facilitates diagnostic objectivity, classification, and prognostication in malignant pleural mesothelioma. Hum Pathol. 2015;46:1670-8. 